\title{
Distribution and Characteristics of Pancreatic Volume Using Computed Tomography Volumetry
}

\author{
Jihyun Yoon ${ }^{1, *}$, Kwang Gi Kim ${ }^{2, *}$, Young Jae Kim², Sangheon Lim$^{2}$, Yeon-Ho Park ${ }^{3}$, Doojin Kim³, \\ Hee-Taik Kang ${ }^{1}$, Doo-Ho Lee ${ }^{3}$ \\ ${ }^{1}$ Department of Family Medicine, Chungbuk National University Hospital, Cheongju, Korea \\ ${ }^{2}$ Department of Biomedical Engineering, Medical Devices R\&D Center, Gachon University Gil Medical Center, Incheon, Korea \\ ${ }^{3}$ Department of Surgery, Gachon University Gil Medical Center, Incheon, Korea
}

Objectives: Changes in the pancreatic volume (PV) are useful as potential clinical markers for some pancreatic-related diseases. The objective of this study was to measure the volume of the pancreas using computed tomography (CT) volumetry and to evaluate the relationships between sex, age, body mass index (BMI), and sarcopenia. Methods: We retrospectively analyzed the abdominal CT scans of 1,003 subjects whose ages ranged between 10 and 90 years. The pancreas was segmented manually to define the region of interest (ROI) based on CT images, and then the PVs were measured by counting the voxels in all ROIs within the pancreas boundary. Sarcopenia was identified by examination of CT images that determined the crosssectional area of the skeletal muscle around the third lumbar vertebra. Results: The mean volume of the pancreas was 62.648 $\pm 19.094 \mathrm{~cm}^{3}$. The results indicated a negative correlation between the PV and age. There was a positive correlation between the PV and BMI for both sexes, females, and males $(r=0.343, p<0.001 ; r=0.461, p<0.001$; and $r=0.244, p<0.001$, respectively). Additionally, there was a positive correlation between the PV and sarcopenia for females $(r=0.253, p<0.001)$ and males $(r=0.200, p<0.001)$. Conclusions: CT pancreas volumetry results may help physicians follow up or predict conditions of the pancreas after interventions for pancreatic-related disease in the future.

Keywords: Pancreas, Deep Learning, Body Mass Index (BMI), Sarcopenia, Computed Tomography (CT)

Submitted: July 26, 2020

Revised: August 2, 2020

Accepted: September 8, 2020

\section{Corresponding Author}

Doo-Ho Lee

Department of Surgery, Gachon University Gil Medical Center, 77421, Namdong-daero, Namdong-gu, Incheon 21565, Korea. Tel: +8232-460-3244, E-mail: dooholeemd@gilhospital.com (https://orcid. org/0000-0002-9939-207X)

*These authors contributed equally to this work.

This is an Open Access article distributed under the terms of the Creative Commons Attribution Non-Commercial License (http://creativecommons.org/licenses/bync/4.0/) which permits unrestricted non-commercial use, distribution, and reproduction in any medium, provided the original work is properly cited.

(C) 2020 The Korean Society of Medical Informatics

\section{Introduction}

Determination of the pancreatic volume (PV) has significant potential for solving clinical problems [1]. Changes in the $\mathrm{PV}$ are related to pathological conditions of the pancreatic endocrine or exocrine function [2]. Thus, the PV can be used as a clinical marker for disease progression [3]. Several studies have indicated that chronic pancreatitis and diabetes reduce the size of pancreas [4], and pancreatic cancer and acute pancreatitis make the pancreas become focal or show diffuse enlargement [1]. The PV can also be used as a predictor of long-term outcomes or the prevalence of organ-specific diseases after resection [5,6]. Therefore, it is necessary to know the normal anatomic range of the PV for defining pathological conditions. 
Computed tomography (CT) has been widely used to noninvasively determine the PV and investigate the relationship between the size of the pancreas and its endocrine and exocrine functions. Previous studies have suggested the average PV using CT $[7,8]$. According to several studies, various clinical parameters affect the PV. The condition of fat deposition in the pancreas has been associated with PV, age, sex, obesity, and dyslipidemia [7,9]. Additionally, sarcopenia is defined as a reduction in the muscle mass and strength, and it is a progressive disease with aging [10], which leads to functional impairment and physical disability. It increases the risk of obesity and is related to fat deposition in the pancreas $[9,11]$. Since sarcopenia is common in patients with pancreatic cancer and is known to affect prognosis after surgical resection and chemotherapy [12], studies on the relationship between sarcopenia and PV are needed. However, studies on the PV have suggested different values [1,4,7-9]. Because most study populations enrolled have been selected from other ethnicities, study of the average PV is needed for Korean populations. In a recent study, it was found that, compared with Caucasians, Koreans had a significantly lower PV and tended to have a higher fat content in the pancreas [13]. However, the small sample sizes in the study on the PV limited the interpretation of the results; a larger number of subjects is needed to clarify the association between the PV and pancreatic function.

In this study, we investigated the normal PV range using CT volumetry for large populations. Additionally, we analyzed the correlations between the PV and gender, age, obesity, and sarcopenia using CT images.

\section{Methods}

\section{Subject Selection}

A total of 1,003 routine health checkup subjects who visited Gachon University Gil Medical Center between January 2017 and February 2020 and underwent abdominal CT scans, were enrolled in this study. The exclusion criterion was the presence of either clinical or CT signs of pancreatic or peri-pancreatic pathology. Additionally, subjects with pathological conditions affecting the PV were excluded, e.g., peritonitis, or a history of chemoradiation due to malignancy in subjects below 19 years of age.

The Institutional Review Board of Gachon University Gil Medical Center approved this study (No. GDIRB2020-121), and no informed consent from the subjects was required. We adhered to the Declaration of Helsinki (1975).

\section{Assessment of PV}

The subjects underwent abdominal-protocol 3-mm-thick three-phase contrast-enhanced axial and coronal CT for the screening program. The pancreas was segmented manually to define the region of interest (ROI) using software that was developed in-house. The ROI was drawn on all axial planes where the pancreas was present, and all ROIs were joined to construct the volume. The PV was determined by multiplying the total number of pixels in all ROIs by the X-axis, Yaxis pixel spacing, and slice thickness within the pancreas boundary.

\section{Assessment of Skeletal Muscle Using CT Images}

The skeletal muscle area around the third lumbar vertebra (L3) region was measured and was selected as the standard landmark, as described in previous studies [14]. We used inhouse software Gachon_DeepBody developed at Gachon University to automatically determine the skeletal muscle area within a range of -25 to 150 Hounsfield units. The Gachon_DeepBody segmented skeletal muscle using the trained deep-learning model and measured the volume. The deep-learning model was trained using the U-Net [14] in CT images of 2,504 cases. The accuracy for skeletal muscle segmentation was $94.87 \%$. Sarcopenia was determined to be present when the L3 skeletal muscle index (SMI) was $\leq 55$ $\mathrm{cm}^{2} / \mathrm{m}^{2}$ for men and $\leq 39 \mathrm{~cm}^{2} / \mathrm{m}^{2}$ for women, according to previously reported cutoff values for the Korean population [14]. L3 SMI was defined as the cross-sectional area of the muscle at the L3 level normalized with respect to the height, as is conventional for the BMI.

\section{Statistical Analysis}

Continuous variables are presented as the mean \pm standard deviation (for normally distributed variables) or as the median and range (for non-normally distributed variables). The continuous variables were compared between the two groups using independent-sample $t$-tests and one-way analysis of variance (ANOVA), and categorical parameters were compared using the $\chi^{2}$ test. The Duncan multiple comparison test was used to compare group means. The subjects were classified into the following groups: underweight (BMI < $\left.18.5 \mathrm{~kg} / \mathrm{m}^{2}\right)$, normal weight $\left(18.5 \leq \mathrm{BMI} \leq 22.9 \mathrm{~kg} / \mathrm{m}^{2}\right)$, overweight $\left(23.0 \leq \mathrm{BMI} \leq 24.9 \mathrm{~kg} / \mathrm{m}^{2}\right)$, and obese (BMI $\geq 25 \mathrm{~kg} /$ $\mathrm{m}^{2}$ ) [15]. All analyses were performed using SPSS Statistics software version 20.0 (IBM Corp., Armonk, NY, USA), and $p<0.05$ was set as the limit for statistical significance. 
Table 1. Baseline characteristics of study population

\begin{tabular}{lccccc}
\hline & Total $(\mathrm{n}=1,003)$ & Female $(\mathrm{n}=474)$ & Male $(\mathrm{n}=529)$ & $t$ & $p$-value \\
\hline Age $(\mathrm{yr})$ & $55.323 \pm 15.621$ & $54.964 \pm 15.980$ & $55.645 \pm 15.300$ & 0.689 & 0.491 \\
Pancreatic volume $\left(\mathrm{cm}^{3}\right)$ & $62.648 \pm 19.094$ & $55.762 \pm 16.064$ & $68.818 \pm 19.493$ & 11.498 & $<0.001$ \\
Height $(\mathrm{cm})$ & $163.580 \pm 8.947$ & $157.000 \pm 6.244$ & $169.000 \pm 6.804$ & 29.515 \\
Weight $(\mathrm{kg})$ & $65.289 \pm 12.146$ & $59.620 \pm 10.018$ & $70.368 \pm 11.635$ & 15.589 \\
BMI $\left(\mathrm{kg} / \mathrm{m}^{2}\right)$ & $24.316 \pm 3.519$ & $24.147 \pm 3.752$ & $24.466 \pm 3.294$ & 1.435 & 0.155 \\
Sarcopenia $(\mathrm{yes})$ & $411(43.97)$ & $269(65.45)$ & $142(34.55)$ & -10.082 & $<0.001$ \\
\hline
\end{tabular}

Values are presented as mean \pm standard deviation or number (\%).

BMI: body mass index.

$p$-values correspond to comparisons between men and women.

Table 2. Change in the pancreatic volume with respect to age

\begin{tabular}{ccccccc}
\hline \multirow{2}{*}{ Age $(\mathrm{yr})$} & $\mathbf{n}$ & \multicolumn{3}{c}{ Pancreatic volume $\left(\mathrm{cm}^{3}\right)$} & \multirow{2}{*}{$\boldsymbol{t}$} & $\boldsymbol{p}$-value \\
\cline { 3 - 6 } & & Total & Female & & \\
\hline $11-20$ & 30 & $64.384 \pm 19.404$ & $61.122 \pm 26.053$ & $66.879 \pm 12.575$ & 0.800 \\
$21-30$ & 53 & $64.642 \pm 15.943$ & $60.361 \pm 13.693$ & $70.225 \pm 17.207$ & 2.325 & 0.024 \\
$31-40$ & 135 & $69.691 \pm 16.147$ & $64.466 \pm 14.652$ & $75.487 \pm 15.847$ & 4.198 & $<0.001$ \\
$41-50$ & 144 & $70.508 \pm 20.413$ & $61.186 \pm 16.737$ & $78.178 \pm 20.053$ & 5.446 & $<0.001$ \\
$51-60$ & 241 & $64.098 \pm 18.766$ & $55.503 \pm 15.357$ & $71.437 \pm 18.337$ & 7.240 & $<0.001$ \\
$61-70$ & 239 & $59.175 \pm 17.977$ & $52.126 \pm 14.043$ & $64.699 \pm 18.819$ & 5.711 & $<0.001$ \\
$71-80$ & 161 & $51.720 \pm 17.085$ & $46.047 \pm 12.365$ & $57.187 \pm 19.176$ & 4.363 & $<0.001$ \\
\hline
\end{tabular}

Values are presented as mean \pm standard deviation.

$p$-values correspond to comparisons between men and women.

\section{Results}

Table 1 shows the baseline characteristics of the subjects, PV, and clinical variables. The mean PV was significantly higher for males $\left(68.818 \pm 19.493 \mathrm{~cm}^{3}\right)$ than for females $(55.762 \pm$ $\left.16.064 \mathrm{~cm}^{3}\right)$. The mean PV values for female and male subjects exhibited a statistically significant difference $(p<0.001)$.

\section{The Relationship between Age and PV}

The PV increases slightly from the early teens to the 40 s and declines thereafter (Table 2, Figure 1). The PV was maximized in the 40s for all groups except for the female group. A significant difference in the PV between females and males was observed for all age groups except for teens.

\section{The Relationship between BMI and PV}

Table 3 presents the mean PV results for each group. A comparison of PVs according to BMI groups revealed significant differences between the normal weight group and the other groups.

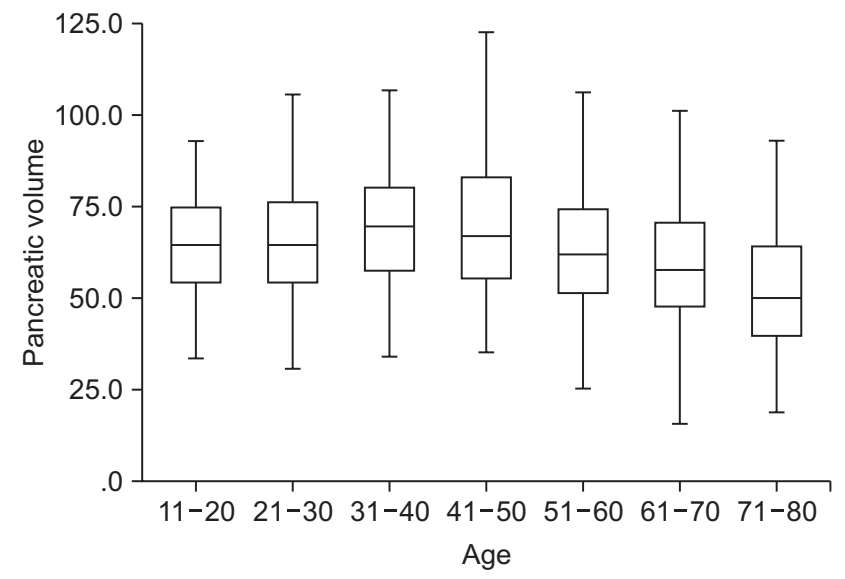

Figure 1. Box-and-whisker plots of pancreatic volume with respect to age. Boxes indicate median and 25th-75th percentile ranges.

\section{The Relationship between Sarcopenia and PV}

As seen in Table 4, the PV was significantly higher for subjects with sarcopenia than for those without sarcopenia ( $p$ $=0.002$ ). The BMI of subjects with sarcopenia was $26.023 \pm$ $3.496 \mathrm{~kg} / \mathrm{m}^{2}$, and the BMI of subjects without sarcopenia was 
Table 3. Relationship between BMI and pancreatic volume

\begin{tabular}{lccccc}
\hline \multicolumn{1}{c}{ Group } & BMI $\left(\mathrm{kg} / \mathrm{m}^{2}\right)$ & $\mathrm{n}$ & Pancreatic volume $\left(\mathrm{cm}^{3}\right)$ & $\mathrm{F}$ & $p$-value \\
\hline Underweight & $<18.5$ & 44 & $46.855 \pm 13.616^{\mathrm{a}}$ & & \\
Normal weight & $18.5-22.9$ & 316 & $57.162 \pm 17.177^{\mathrm{b}}$ & 33.874 & $<0.001$ \\
Overweight & $23.0-24.9$ & 248 & $63.249 \pm 18.433^{\mathrm{c}}$ & \\
Obese & $\geq 25.0$ & 395 & $68.419 \pm 19.299^{\mathrm{d}}$ & \\
\hline
\end{tabular}

Values are presented as mean \pm standard deviation.

BMI: body mass index.

Statistical significances were tested by one-way analysis of variances among groups.

The superscripts indicate significant difference between groups based on Duncan multiple comparison test.

Table 4. Relationship between sarcopenia and pancreatic volume

\begin{tabular}{|c|c|c|c|c|}
\hline & Normal muscle mass $(n=592)$ & Sarcopenia $(n=411)$ & $t$ & $p$-value \\
\hline Weight (kg) & $63.660 \pm 11.266$ & $67.633 \pm 12.970$ & -5.157 & $<0.001$ \\
\hline Height $(\mathrm{cm})$ & $165.537 \pm 8.422$ & $160.757 \pm 8.937$ & 8.621 & $<0.001$ \\
\hline BMI $\left(\mathrm{kg} / \mathrm{m}^{2}\right)$ & $23.130 \pm 3.014$ & $26.023 \pm 3.450$ & -13.994 & $<0.001$ \\
\hline Underweight & $36(6.1)$ & $8(1.9)$ & & \\
\hline Normal weight & $256(43.2)$ & $60(14.6)$ & & \\
\hline Overweight & $147(24.8)$ & $101(58.9)$ & & \\
\hline Obesity & $153(25.8)$ & $242(58.9)$ & & \\
\hline Gender & & & -10.082 & $<0.001$ \\
\hline Female & 205 & 269 & & \\
\hline Male & 387 & 142 & & \\
\hline Age (yr) & $56.534 \pm 15.665$ & $53.579 \pm 15.410$ & 2.957 & 0.003 \\
\hline Pancreatic volume $\left(\mathrm{cm}^{3}\right)$ & $61.070 \pm 19.086$ & $64.921 \pm 18.898$ & -3.155 & 0.002 \\
\hline
\end{tabular}

Values are presented as mean \pm standard deviation or number $(\%)$.

BMI: body mass index.

Table 5. Association between clinical variables studied and pancreatic volume

\begin{tabular}{|c|c|c|c|c|c|c|}
\hline & \multicolumn{6}{|c|}{ Pancreatic volume } \\
\hline & \multicolumn{2}{|c|}{ Total $(n=1,003)$} & \multicolumn{2}{|c|}{ Female $(n=529)$} & \multicolumn{2}{|c|}{ Male $(n=474)$} \\
\hline & $r$ & $p$-value & $r$ & $p$-value & $r$ & $p$-value \\
\hline Age & -0.274 & $<0.001$ & -0.259 & $<0.05$ & -0.357 & $<0.001$ \\
\hline Weight & 0.532 & $<0.001$ & 0.524 & $<0.05$ & 0.34 & $<0.001$ \\
\hline Height & 0.428 & $<0.001$ & 0.297 & $<0.05$ & 0.265 & $<0.001$ \\
\hline BMI & 0.343 & $<0.001$ & 0.461 & $<0.05$ & 0.224 & $<0.001$ \\
\hline Sarcopenia & 0.099 & $<0.001$ & 0.253 & $<0.05$ & 0.2 & $<0.001$ \\
\hline
\end{tabular}

$r$ is Pearson correlation coefficient.

$23.130 \pm 3.0143 \mathrm{~kg} / \mathrm{m}^{2}$

\section{Association between Clinical Variables and PV}

As seen in Table 5, the Pearson correlation analysis revealed a statistically significant correlation between the parameters used and the PV. For all three groups, the PV was positively correlated with weight and height. There was a negative correlation between the PV and age for both sexes $(r=-0.274$, $p<0.001)$, females $(r=-0.259, p<0.001)$, and males $(r=$ $-0.357, p<0.001)$. We found a statistically significant correla- 
tion between the PV and BMI for both sexes, females, and males $(r=0.343, p<0.001 ; r=0.461, p<0.001$; and $r=0.224$, $p<0.001$, respectively). There was also a statistically positive relationship between sarcopenia and the PV $(p=0.002)$.

\section{Discussion}

In this study, we investigated the PV range for healthy subjects and the relationship between the PV and clinical variables, namely, sex, age, BMI, and sarcopenia.

We found that the mean PV for 1,003 individuals was $62.648 \pm 19.094 \mathrm{~cm}^{3}$. Various studies have been performed on the PV $[1,4,8,9,13,16-18]$. For example, Goda et al. [4] reported values in the range of $71.5 \pm 18.7 \mathrm{~cm}^{3}$ for 22 individuals (mean age of 46 years), Geraghty et al. [8] reported values in the range of $64.4 \pm 18.1 \mathrm{~cm}^{3}$ for 46 female individuals and $87.4 \pm 21.3 \mathrm{~cm}^{3}$ for 57 male individuals (mean ages of 49 and 48 years, respectively). The discrepancies in the mean PV compared with those from previous studies may be due to differences in the number, average age, anthropometric characteristics, and genders of the individuals enrolled in the study and differences in the ethnicities between the study populations.

We found a statistically significant correlation between the $\mathrm{PV}$ and the age of the participants in the present study. For healthy people, the PV increased with age, and it started to decline after 50 years, which is consistent with the findings of previous studies [16,17].

We found that the mean PV based on CT was $55.76 \mathrm{~cm}^{3}$ for females and $68.82 \mathrm{~cm}^{3}$ for males. The mean PV was $19.0 \%$ larger for males than for females, which is consistent with the findings of previous anatomical and radiological studies $[1,8,9,16,19]$. However, the PV obtained in this study is smaller than those of other studies involving Korean individuals [13]. This could be attributed to differences in the BMI $\left(26.1 \mathrm{~kg} / \mathrm{m}^{2}\right.$ in the study of Lim et al. [13] vs. $24 \mathrm{~kg} / \mathrm{m}^{2}$ in our study), the number of subjects enrolled, and differences in the anthropometric characteristics.

There was a correlation between the PV and BMI $(r=0.343$, $p<0.05)$, and these results were consistent with previous reports $[9,17]$. The correlation suggests that obesity affects the PV. The fact that the PV was greater in obese people may be due to an increase in the amount of pancreatic fat rather than in pancreatic parenchyma [9]. The incidence of fatty replacement, which is also known as lipomatosis, is associated with obesity and DM [11,17].

Additionally, there was a statistically significant correlation between the PV and sarcopenia. In this study, approximately
$98 \%$ of the subjects had sarcopenia and were of normal weight or overweight/obese, and only $2 \%$ of the subjects had sarcopenia and were underweight. This indicates the limitation of body-composition evaluation based on BMI. BMI, which is based on the overall body mass, does not differentiate between fat mass and muscle mass [20].

In our study, sarcopenia was measured using CT, which allowed for the determination of changes in the skeletal muscle mass. Sarcopenia is defined as a reduction in the muscle mass and strength and is a progressive disease related to frailty in elderly subjects [21]. Sarcopenia is related to fat deposition in the pancreas as well as in muscle [22]. In the present study, the mean age of subjects with sarcopenia was 53 years, and sarcopenia was present in $41 \%$ of the subjects with a larger BMI. We also considered the concept of sarcopenic obesity [23], which occurs with aging and may lead to an increased risk of both sarcopenia and obesity. The subjects with sarcopenia in our study were obese, which is consistent with the findings of a previous study, indicating that the PV is correlated with a high BMI [24].

In contrast, some studies have indicated that the $\beta$-cell function in the pancreas is suppressed with aging [25], reducing the PV. For example, in a study on patients with type I diabetes, the PV was lower for insulin-dependent diabetic patients than for normal subjects [26], suggesting that reduced PV is associated with $\beta$-cell dysfunction. Additionally, a study on the association between sarcopenia and the clinical parameters of $\beta$-cell function and insulin resistance revealed that reduced $\beta$-cell function is associated with reduced skeletal muscle mass in subjects without diabetes [27]. We used CT images to measure the PV; thus, pathological changes, such as reduced $\beta$-cell function in the pancreas, could not be directly evaluated. However, CT allows accurate noninvasive measurement of the PV in vivo, as well as parameters that affect the PV. A recent study on patients undergoing pancreatoduodenectomy indicated that PV measurement via CT was useful for evaluating the pancreatic endocrine function [28].

Our study had several limitations. First, we measured the PV using CT images. Thus, confounding factors (e.g., patients with conditions such as prediabetes, diabetes, and hypertension; contrast medium factors; and CT scanning factors) may have influenced the PV analysis. Second, because the study was conducted at a single tertiary care hospital, the results cannot be extrapolated to the entire population of Korea. Third, we did not measure endocrine cell function in the pancreas. Additionally, because this was a cross-sectional study, we cannot explain the sequential relationship between 
the PV and the individual-matched variables used in this study. Additionally, sarcopenia is known to be related to aging. In our study, we found that subjects in their teens were diagnosed with sarcopenia using CT images. Therefore, it might be difficult to understand all the comorbidities for the subjects.

The present study had several strengths. First, the results may be more reliable than those of previous studies because a large number of subjects were examined, which may have improved the significance and clinical value of the findings. Second, there were previously no studies in which the PV was correlated with sarcopenia.

In summary, we found a positive correlation between BMI and the PV in a large number of Koreans, which is consistent with the findings of previous studies. Additionally, we found a relationship between sarcopenia and the PV. This study may be useful for the early diagnosis of sarcopenia using CT scans and the prevalence of new-onset diabetes after surgery for pancreatic diseases.

\section{Conflict of Interest}

Kwang Gi Kim is an editor of Healthcare Informatics Research; however, he did not involve in the peer reviewer selection, evaluation, and decision process of this article. Otherwise, no potential conflict of interest relevant to this article was reported.

\section{Acknowledgments}

This work was supported by Institute for National IT Industry Promotion Agency (NIPA) grant funded by the Korea government (MSIT) (No. A0602-19-1032, Intelligent surgical guide system \& service from surgery video data analytics), and the Gachon University Gil Medical Center (No. FRD2019-16).

\section{ORCID}

Jihyun Yoon (http://orcid.org/0000-0002-1057-2235)

Kwang Gi Kim (http://orcid.org/0000-0001-9714-6038)

Young Jae Kim (http://orcid.org/0000-0003-0443-0051)

Sangheon Lim (http://orcid.org/0000-0002-1298-5180)

Yeon-Ho Park (http://orcid.org/0000-0003-1623-2167)

Doojin Kim (http://orcid.org/0000-0002-2382-6724)

Hee-Taik Kang (http://orcid.org/0000-0001-8048-6247)

Doo-Ho Lee (http://orcid.org/0000-0002-9939-207X)

\section{References}

1. Djuric-Stefanovic A, Masulovic D, Kostic J, Randjic K, Saranovic D. CT volumetry of normal pancreas: correlation with the pancreatic diameters measurable by the cross-sectional imaging, and relationship with the gender, age, and body constitution. Surg Radiol Anat 2012;34(9):811-7.

2. Saisho Y. Pancreas volume and fat deposition in diabetes and normal physiology: consideration of the interplay between endocrine and exocrine pancreas. Rev Diabet Stud 2016;13(2-3):132-47.

3. Williams AJ, Thrower SL, Sequeiros IM, Ward A, Bickerton AS, Triay JM, et al. Pancreatic volume is reduced in adult patients with recently diagnosed type 1 diabetes. J Clin Endocrinol Metab 2012;97(11):E2109-13.

4. Goda K, Sasaki E, Nagata K, Fukai M, Ohsawa N, Hahafusa T. Pancreatic volume in type 1 und type 2 diabetes mellitus. Acta diabetologica 2001;38:145-9.

5. Miyamoto R, Oshiro Y, Sano N, Inagawa S, Ohkohchi $\mathrm{N}$. Remnant pancreatic volume as an indicator of poor prognosis in pancreatic cancer patients after resection. Pancreatology 2019;19(5):716-21.

6. Hirata K, Nakata B, Amano R, Yamazoe S, Kimura K, Hirakawa K. Predictive factors for change of diabetes mellitus status after pancreatectomy in preoperative diabetic and nondiabetic patients. J Gastrointest Surg 2014;18(9):1597-603.

7. Caglar V, Kumral B, Uygur R, Alkoc OA, Ozen OA, Demirel H. Study of volume, weight and size of normal pancreas, spleen and kidney in adults autopsies. Forensic Med Anat Res 2014;2:63-9.

8. Geraghty EM, Boone JM, McGahan JP, Jain K. Normal organ volume assessment from abdominal CT. Abdom Imaging 2004;29(4):482-90.

9. Saisho Y, Butler AE, Meier JJ, Monchamp T, AllenAuerbach M, Rizza RA, et al. Pancreas volumes in humans from birth to age one hundred taking into account sex, obesity, and presence of type- 2 diabetes. Clin Anat 2007;20(8):933-42.

10. Lee JS, Kim YS, Kim EY, Jin W. Prognostic significance of CT-determined sarcopenia in patients with advanced gastric cancer. PLoS One 2018;13(8):e0202700.

11. Matsuda Y. Age-related pathological changes in the pancreas. Front Biosci (Elite Ed) 2018;10:137-42.

12. Chan MY, Chok KS. Sarcopenia in pancreatic cancer: effects on surgical outcomes and chemotherapy. World J Gastrointest Oncol 2019;11(7):527-37. 
13. Lim S, Bae JH, Chun EJ, Kim H, Kim SY, Kim KM, et al. Differences in pancreatic volume, fat content, and fat density measured by multidetector-row computed tomography according to the duration of diabetes. Acta Diabetol 2014;51(5):739-48.

14. Ronneberger O, Fischer P, Brox T. U-Net: convolutional networks for biomedical image segmentation. In: Navab N, Hornegger J, Wells W, Frangi A, editors. Medical image computing and computer-assisted intervention. Cham, Switzerland: Springer; 2015. p. 234-41.

15. WHO Expert Consultation. Appropriate body-mass index for Asian populations and its implications for policy and intervention strategies. Lancet 2004;363(9403):15763.

16. Kou K, Saisho Y, Jinzaki M, Itoh H. Relationship between body mass index and pancreas volume in Japanese people. JOP 2014;15(6):626-7.

17. Caglar V, Songur A, Yagmurca M, Acar M, Toktas M, Gonul Y. Age-related volumetric changes in pancreas: a stereological study on computed tomography. Surg Radiol Anat 2012;34(10):935-41.

18. Mu'ti A, Paramita S. Relationship of pancreatic volumes using CT scan in indonesian adults with age, sex, and body mass index. Folia Medica Indonesiana 2020;56(1):31-5.

19. de la Grandmaison GL, Clairand I, Durigon M. Organ weight in 684 adult autopsies: new tables for a Caucasoid population. Forensic Sci Int 2001;119(2):149-54.

20. Prado CM, Heymsfield SB. Lean tissue imaging: a new era for nutritional assessment and intervention. JPEN J
Parenter Enteral Nutr 2014;38(8):940-53.

21. Cruz-Jentoft AJ, Bahat G, Bauer J, Boirie Y, Bruyere O, Cederholm T, et al. Sarcopenia: revised European consensus on definition and diagnosis. Age Ageing 2019;48(1):16-31.

22. Zamboni M, Gattazzo S, Rossi AP. Myosteatosis: a relevant, yet poorly explored element of sarcopenia. Eur Geriatr Med 2019;10(1):5-6.

23. Baumgartner RN. Body composition in healthy aging. Ann N Y Acad Sci 2000;904:437-48.

24. Butler AE, Janson J, Bonner-Weir S, Ritzel R, Rizza RA, Butler PC. Beta-cell deficit and increased betacell apoptosis in humans with type 2 diabetes. Diabetes 2003;52(1):102-10.

25. Oya J, Nakagami T, Yamamoto Y, Fukushima S, Takeda $\mathrm{M}$, Endo Y, et al. Effects of age on insulin resistance and secretion in subjects without diabetes. Intern Med 2014;53(9):941-7.

26. Sakata N, Egawa S, Rikiyama T, Yoshimatsu G, Masuda $\mathrm{K}$, Ohtsuka $\mathrm{H}$, et al. Computed tomography reflected endocrine function of the pancreas. J Gastrointest Surg 2011;15(3):525-32.

27. Sakai, S, Tanimoto K, Imbe A, Inaba Y, Shishikura K, Tanimoto $Y$, et al. Decreased $\beta$-cell function is associated with reduced skeletal muscle mass in Japanese subjects without diabetes. PloS One 2016;11(9):e0162603.

28. Balzano G, Dugnani E, Gandolfi A, Scavini M, Pasquale V, Aleotti F, et al. Effect of diabetes on survival after resection of pancreatic adenocarcinoma: a prospective, observational study. PLoS One 2016;11(11):e0166008. 